\title{
Reduced symmetry and analogy to chirality in periodic dielectric media
}

\author{
I. H. Giden \\ igiden@etu.edu.tr
}

M. Turduev

H. Kurt

\begin{abstract}
Nanophotonics Research Laboratory, Department of Electrical and Electronics Engineering, TOBB University of Economics and Technology, Ankara, 06560 Turkey

Nanophotonics Research Laboratory, Department of Electrical and Electronics Engineering, TOBB University of Economics and Technology, Ankara, 06560 Turkey

Nanophotonics Research Laboratory, Department of Electrical and Electronics Engineering, TOBB University of Economics and Technology, Ankara, 06560 Turkey
\end{abstract}

Much attention has been paid to photonic applications based on periodic media. Meanwhile, quasi-periodic and disordered media have extended the research domain and provided additional novelties for manipulating and controlling light propagation. This review article attempts to highlight the benefits of symmetry reduction in highly symmetric periodic photonic media, and applies the concept of chirality to all-dielectric materials arranged in special orders. Two-dimensional periodic structures known as photonic crystals (PCS) are highly symmetric in terms of structural patterns, due to the lattice types and shape of the elements occupying the PC unit-cell. We propose the idea of intentionally introducing reduced-symmetry, to search for anomalous optical characteristics so that these types of PCs can be used in the design of novel optical devices. Breaking either translational or rotational symmetries of PCs provides enhanced and additional optical characteristics such as creation of a complete photonic bandgap, wavelength demultiplexing, super-collimation, tilted self-collimation, and beam deflecting/routing properties. Utilizing these characteristics allows the design of several types of photonic devices such as polarization-independent waveguides, wavelength demultiplexers, beam deflectors, and routers. Moreover, reducing the symmetry in the PC unit-cell scale produces a novel feature in all-dielectric PCS that is known as chirality. On the basis of above considerations, it is expected that low-symmetric PCS can be considered as a potential structure in photonic device applications, due to the rich inherent optical properties, providing broadband operation, and being free of absorption losses.

[DOI: http://dx.doi.org/10.2971/jeos.2014.14045i]

Keywords: Photonic crystals, non-periodic photonic media, tilted self-collimation, super-collimation, beam deflection, beam routers, beam splitters, wavelength demultiplexing, polarization-insensitive devices, chirality, all-dielectric medium

\section{INTRODUCTION TO PHOTONIC CRYSTALS WITH REDUCED-SYMMETRY}

In order to exploit novel optical properties of light, it is essential to convert a medium with a homogeneous refractive index into a periodically modulated one. One-dimensional configurations with small refractive index contrast are the most popular structures in optical devices and have been used for a long time. The pioneering study on multi-layered and periodic dielectric structures was conducted by Rayleigh in 1887 [1]. That study showed that it is possible to find a photonic band gap (PBG) in one-dimensional periodic structures. Integrated versions of one-dimensional periodic structures have been investigated and used in photonic devices such as multi-layered dielectric mirrors, Bragg gratings, distributed feedback lasers, and vertical cavity surface emitting lasers [2]. The common property of such devices is a small refractive index contrast modulated along one dimension. Hundred years later, in 1987, Yablonovitch and John suggested a new type of dielectric structure called photonic crystals (PCs) that may be multidimensional periodic and possess a high index contrast ratio [3, 4]. Such nanoscale electromagnetic band gap materials are called photonic, since strong photon interactions occur with these types of periodic structures. One of the basic characteristics of PCs is that the refractive index variation may appear in one, two, or even three-dimensions. The PC concept has been extensively studied in the photonics field since 1987 because of its ability to control the flow of light. One of the most attractive aspects of PCs is that the light-matter interaction in PCs enables unique optical conditions that cannot be observed in standard optical waveguides, e.g., slowlight, graded-index PC design, optical cavities with high Qfactor, super-prisms, self-collimators, sensitive bio-chemical PC based sensors, specific light sources, and lasers [5]-[16]. Furthermore, light motion inside PCs can be analyzed by scale-invariant Maxwells equations, so that structural PC unitcell parameters can be easily tuned either to millimeter or micron-scale [17]-[20].

Meanwhile, research into aperiodic and disordered PC structures has attracted the much attention [21]. The interaction of photons with these types of structures allows exciting optical phenomena to be obtained; light scattering in disordered media may provide strong photon localization [22]. Disordered structures have potential in some applications such as random lasing, Anderson localization, sub-wavelength imaging, and novel light-source designs [23]. In a recent work, a compact spectrometer with high reso- 
lution was designed by intentionally introducing disorder into the photonic medium [24]. Utilizing random gain medium for lasing action is another research topic that exploits light scattering and amplification in disordered materials [24]-[28].

Periodic structures may be disadvantageous in some cases because of their high-symmetry. For example, high-symmetric structures are very sensitive to structural deformation. Moreover, the operating bandwidth may be quite small for the high-symmetric PC case. Besides, structural degradation during the fabrication process can be considered as another possible problem, since it causes deviation from the ideal cases. Lastly, unusual optical characteristics may be expected while reducing the symmetry of PC structures.

In addition to periodic and disordered PC configurations, quasi-crystals are a topic of much interest and have been intensively studied. Translational symmetry is broken in quasi-periodic structures, whereas rotational symmetry is kept intact [29]. Although random and disordered PCs do not have any spatial symmetry property, quasi-periodic structures possess a reduced symmetry characteristic; these types of periodic structures have high rotational symmetry and, therefore, anomalous characteristics may arise, especially in transmission spectra and photonic band gaps [30]-[33]. Due to the high rotational symmetries of quasi-crystals, their forbidden band gaps and light transport properties are superior to regular PCs [34]-[40]. Furthermore, using these types of PC designs, unique properties appear in transmission, reflection, refraction, localization, radiation of photons, symmetry in Fourier space, nonlinear optical, and diffraction characteristics. For example, enhancement of the light radiation in polymer lightemitting diodes has been achieved by using quasi-periodic PC structures [41].

In this study, low-symmetric PCs, which are different types of PCs to those mentioned above, will be presented and reviewed. What we refer to by low-symmetric or reducedsymmetry PCs is that, in principle, either rotational or translational symmetry is broken in the PC unit-cell. The rotational symmetry in PCs can be broken by locating more than one PC rod at different positions in the PC unit-cell. Duplication of such a unit-cell in certain directions creates a periodic structure, either in a square or triangular-lattice form. On the other hand, discrete translational symmetry holds in PCs when they are periodic, and in that case, the eigenmodes for PC structure can be identified by Bloch-Floquet states [42]. The translational symmetry property can be broken by introducing defects/cavities into the structure and constructing disordered or quasi-periodic PC lattices [43].

Limited attempts were made to investigate low symmetric PCs solely for enhanced photonic band gap properties [44]-[51]. Either the lattice type or scatterer shape was manipulated to produce an enlarged photonic band gap. Forbidden band gap features can be utilized for waveguide and cavity designs by using low symmetry PCs. Such an approach has not been widely discussed in the literature. We know that PCs do not only work under photonic band gap and defect mode scenarios; defect free versions of the designs also yield novel light propagation characteristics. Moreover, the limited low symmetry studies rarely promote photonic modes interacting with such structures.

Band diagram engineering has been performed, paying special attention to enlarging the gap opening and maximizing the gaps overlap. On the other hand, both band movements (slope and form change of the dispersion curves) and degeneracy point splitting, at the symmetry points of the irreducible Brillouin zone, occur depending on symmetry-reduction in PC unit-cell. Iso-frequency contour engineering is an additional mechanism to inspect photonic periodic structures. For the low symmetry unit-cells, iso-frequency contours may indicate unique optical properties for photons. Symmetry reduced photonic media have great potential for several important concepts such as light propagation, reflection, refraction, slow-light, diffraction-free beam propagation, and wavelength de-multiplexing. In the present review, we discuss recent progress in the field by referencing papers, mainly by the current authors, and speculate on feasible, future research directions.

The symmetry reduction in our case is achieved by introducing either more than one piece of dielectric or a complex shaped element, and choosing appropriate locations for them inside the unit-cell. Then it is possible to implement a unitcell such that the mirror image of the unit-cell is not superposable. This is similar to a chiral molecule in chemistry that has a non-superposable mirror image. A materials chirality provides comprehensive information on its stereochemistry. The spatial arrangement of the atoms of molecules and how this affects the physical and chemical properties of the species plays an important role in stereochemistry. Different structural arrangements of atoms may demonstrate different chemical characteristics, even with the same molecular formula. As a result, motivated by the concept of chirality in chemistry, we intend to use PCs with chiral unit-cells. We expect to obtain novel light propagation characteristics in such photonic structures.

The rest of the paper is organized as follows: In Section 2, we provide symmetry definitions in PCs. Then, an analytical approach for the calculation of the band-structure of lowsymmetric PCs is presented in Section 3. We introduce a new concept by making an analogy to chirality in Section 4 . The last two sections (Sections 5 and 6) include discussions of potential applications, future research directions, and the conclusions.

\section{SYMMETRY DEFINITION IN PHOTONIC CRYSTALS}

Square lattice PCs have translational symmetry with respect to lattice vectors, $\mathbf{a}_{1}$ and $\mathbf{a}_{2}$, so that the dielectric permittivity of the periodic structure can be defined by, $\varepsilon(\mathbf{r})=\varepsilon\left(\mathbf{r}+l \mathbf{a}_{1}+m \mathbf{a}_{2}\right)$, in which $l$ and $m$ are integers. As shown in Figure 1(a), for the square lattice PC cylinders, the corresponding lattice unit vectors are, $\mathbf{a}_{1}=a \hat{x}$ and $\mathbf{a}_{2}=a \hat{y}$, 


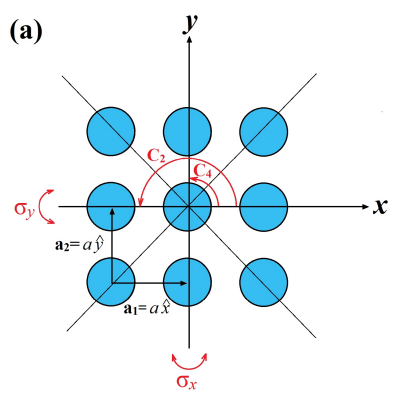

(b)

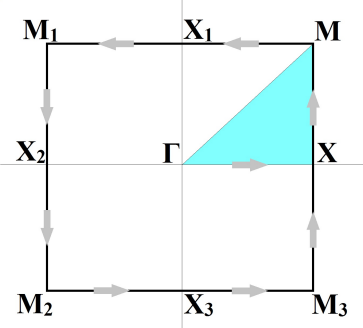

FIC. 1 (a) Symmetry operations for the square-lattice PCs. (b) The corresponding Brillouin zone.

where $\hat{x}$ and $\hat{y}$ are the base vectors in the spatial domain and $a$ is the lattice constant. In addition to translational symmetry, two-dimensional PCs may have other types of symmetries such as mirror and rotational symmetries. If the PC design is invariant under the mirror reflection along the $x$-axis by the operation, $\sigma_{x}$, then the corresponding dielectric constant function does not change depending on sign of $x$, i.e., $\varepsilon(x, y)=\varepsilon(-x, y)$. Similarly, if the PC structure has a mirror symmetry under an operation, $\sigma_{y}$, then the dielectric permittivity function is invariant to the change of sign $y$, $\varepsilon(x, y)=\varepsilon(x,-y)$. The mirror symmetry operators, $\sigma_{x}$ and $\sigma_{y}$, are given as insets in Figure 1(a). The rotational symmetry operation is another symmetry operation to be considered. It is denoted by $C_{n}$, which means the PC structure can be rotated by $\frac{2 \pi}{n}$ radian in a counterclockwise direction about the origin without altering its geometry [52]. Figure 1(a) shows schematic representations of two examples of rotational symmetry operations, namely $C_{2}$ and $C_{4}$.

The band structure of a crystal provides significant information about its optical properties. When the PC lattice has rotational or mirror symmetry, then the band structures also have that symmetry [52]. In such a case, we do not need to consider every $\mathrm{k}$ point in the Brillouin zone. The smallest region within the Brillouin zone is called the Irreducible Brillouin zone, where the symmetries in frequency bands cannot be taken into account. Figure 1(b) shows a schematic diagram of the first Brillouin zone of the square lattice PC, in which the Irreducible Brillouin zone is represented by the shaded region. On the other hand, when either the mirror or rotational symmetry of the structure is broken at a unit-cell scale, by reducing the symmetry of PC rods, the photonic band calculations in the Irreducible Brillouin zone are not sufficient anymore. Instead, every k- point at the edges of first Brillouin zone should be considered, and, thus, the band structure of low-symmetric PCs should be calculated along the $\left[\Gamma-X-M-X_{1}-M_{1}-X_{2}-M_{2}-X_{3}-M_{3}-X-\Gamma\right]$ path, which is shown by the arrows in Figure 1(b). In such a lowsymmetric PC case, maxima and minima of photonic bands at high-symmetry points in the Brillouin zone may shift accordingly, which results in the variation of the band gap boundaries [53].

The detailed analytical investigation for the reduced symmetry PCs will be conducted in the next section.

\section{AN ANALYTICAL APPROACH TO THE BAND STRUCTURE OF LOW SYMMETRIC PHOTONIC CRYSTALS}

An analytical approach can be made in order to determine band-structure of periodic PCs as well as to better understand how the band structure is affected when a low-symmetry is introduced to the PC unit-cell. For that purpose, one should consider the well-known time-dependent Maxwells equations in source-free dielectric media:

$$
\begin{aligned}
\nabla \cdot \mathbf{H}(\mathbf{r}, t) & =0, \quad \nabla \times \mathbf{H}(\mathbf{r}, t)-\varepsilon(\mathbf{r}) \frac{\partial \mathbf{E}(\mathbf{r}, t)}{\partial t}=0, \\
\nabla \cdot \varepsilon(\mathbf{r}) \mathbf{E}(\mathbf{r}, t) & =0, \quad \nabla \times \mathbf{E}(\mathbf{r}, t)+\mu_{0} \frac{\partial \mathbf{H}(\mathbf{r}, t)}{\partial t}=0,
\end{aligned}
$$

where $\mathbf{H}$ and $\mathbf{E}$ represent the magnetic and electric fields, respectively, depending on the coordinates in space and time, $(\mathbf{r}, t)$. Moreover, $\varepsilon$ and $\mu_{0}$ are the position-dependent dielectric permittivity and the permeability in free space, respectively. Time-harmonic $\mathbf{E}(\mathbf{r}, t)$ and $\mathbf{H}(\mathbf{r}, t)$ field vectors can be represented in terms of the vector field phasors, $\mathbf{E}(\mathbf{r})$ and $\mathbf{H}(\mathbf{r})$, such that

$$
\mathbf{E}(\mathbf{r}, t)=\mathbf{E}(\mathbf{r}) \exp (-i \omega t), \quad \mathbf{H}(\mathbf{r}, t)=\mathbf{H}(\mathbf{r}) \exp (-i \omega t),
$$

in which $\omega$ is the angular frequency. Then, Maxwell's equation for the steady state can be written in terms of the vector field phasors as follows:

$$
\begin{gathered}
\nabla \cdot \mathbf{H}(\mathbf{r})+i \omega(\varepsilon(\mathbf{r}) \mathbf{E}(\mathbf{r}))=0 \\
\nabla \cdot \mathbf{E}(\mathbf{r})-i \omega\left(\mu_{0} \mathbf{H}(\mathbf{r})\right)=0
\end{gathered}
$$

In dielectric media, Maxwells equation can be expressed in terms of only the magnetic field phasor, $\mathbf{H}(\mathbf{r})$, as follows:

$$
\nabla \times\left[\frac{1}{\varepsilon(\mathbf{r})} \nabla \times \mathbf{H}(\mathbf{r})\right]=\left(\frac{\omega}{c_{0}}\right)^{2} \mathbf{H}(\mathbf{r}) .
$$

This equation is called the Master equation and the term $c_{0}$ is the phase velocity of wave propagation in vacuum. Thus, the Master equation can be expressed in terms of an eigenvalue problem, and according to Bloch's theorem [42], the electromagnetic field in periodic media can be expanded into a set of harmonic (Bloch) modes, which must satisfy the following relations:

$$
\mathbf{H}(\mathbf{r})=\mathbf{H}_{k}(\mathbf{r}) \exp (i \mathbf{k} \cdot \mathbf{r})
$$

where $\mathbf{H}_{k}(\mathbf{r})$ is a periodic function of the lattice structure, i.e., $\mathbf{H}_{k}(\mathbf{r})=\mathbf{H}_{k}(\mathbf{r}+\mathbf{R})$, where $\mathbf{R}$ is the lattice vector and $\mathbf{k}$ is the Bloch wavevector in the first Brillouin zone. The methodology used for the following derivations is similar to one used previously [54]. Using the Fourier transformation, the magnetic field, $\mathbf{H}_{k}(\mathbf{r})$, and the dielectric function, $\varepsilon(\mathbf{r})$, can be expressed as the sum of plane waves,

$$
\begin{aligned}
\mathbf{H}_{k}(\mathbf{r}) & =\sum_{\mathbf{G}} \sum_{\mathbf{j}=1,2,3, \ldots} \hat{e}_{j} H_{j, \mathbf{k}} \exp (i \mathbf{G} \cdot \mathbf{r}), \\
\varepsilon(\mathbf{r}) & =\sum_{\mathbf{G}} \varepsilon(\mathbf{G}) \exp (i \mathbf{G} \cdot \mathbf{r}),
\end{aligned}
$$

where $\hat{e}_{j}$ denotes the unit vector for the magnetic field and $\mathbf{G}$ is the reciprocal lattice vector. Then, substituting the expansions 
from Eqs. (7) and (8) into the Master equation, Eq. (5), one can obtain two equations for two-dimensional periodic lattices,

$$
\begin{aligned}
& \sum_{\mathbf{G}^{\prime}}|\mathbf{k}+\mathbf{G}|\left|\mathbf{k}+\mathbf{G}^{\prime}\right| \varepsilon^{-1}\left(\mathbf{G}+\mathbf{G}^{\prime}\right) H_{\perp, \mathbf{k}} \mathbf{G}^{\prime}=\left(\frac{\omega}{c_{0}}\right)^{2} H_{\perp, \mathbf{k}} \mathbf{G}, \\
& \sum_{\mathbf{G}^{\prime}}(\mathbf{k}+\mathbf{G})\left(\mathbf{k}+\mathbf{G}^{\prime}\right) \varepsilon^{-1}\left(\mathbf{G}+\mathbf{G}^{\prime}\right) H_{\|, \mathbf{k}} \mathbf{G}^{\prime}=\left(\frac{\omega}{c_{0}}\right)^{2} H_{\|, \mathbf{k}} \mathbf{G},
\end{aligned}
$$

for transverse-magnetic $\left(T M, H_{x}, H_{y}, E_{z}\right)$ and transverseelectric (TE, Ex, Ey, Hz) polarizations. In Eqs. (9) and (10), $\varepsilon^{-1}\left(\mathbf{G}-\mathbf{G}^{\prime}\right)$ is the inverse matrix of $\varepsilon\left(\mathbf{G}-\mathbf{G}^{\prime}\right)$ and the subscripts $\|$ and $\perp$ stand for the directions parallel and perpendicular to the $z$-axis. The dielectric permittivity matrix can be calculated as:

$$
\varepsilon(\mathbf{G})=\frac{1}{A} \int_{A} \varepsilon(\mathbf{r}) \exp (-i \mathbf{G} \cdot \mathbf{r}) d \mathbf{r},
$$

in which $A$ is the area per PC unit-cell and the integral is taken over that area. Eq. 11 may also be simplified in such a way that,

$$
\varepsilon(\mathbf{G})=\left\{\begin{array}{cc}
f \varepsilon_{d}+(1-f) \varepsilon_{b} & \text { for } \quad \mathbf{G}=0, \\
\left(\varepsilon_{d}-\varepsilon_{b}\right) S(\mathbf{G}) & \text { for } \quad \mathbf{G} \neq 0,
\end{array}\right.
$$

where $\varepsilon_{d}$ and $\varepsilon_{b}$ refer to the dielectric constants of the cylindrical PC rods and background and $f$ is the corresponding filling factor. The function, $S(\mathbf{G})$, is the structure factor and it depends directly on the geometry of dielectric region in the PC unit-cell. It is given by the following relation:

$$
S(\mathbf{G})=\frac{1}{A} \int_{A} \exp (-i \mathbf{G} \cdot \mathbf{r}) d \mathbf{r},
$$

where the integration is taken over one PC unit-cell. From the relations given above, it can be understood that while the dielectric distribution in the PC unit-cell, $\varepsilon(\mathbf{r})$, is modified, then $S(\mathbf{G})$ is directly influenced; therefore, by solving Eqs. (9) and (10), the investigated band structures in either TM or TE polarizations may become completely different. To clarify our discussion, we applied the above formulas for different types of square lattice PC configurations and calculated the corresponding structure factor, $S(\mathbf{G})$.

As can be seen in Figure 2(a), in the square lattice case with a lattice constant, $a$, the corresponding lattice vectors are, $\mathbf{a}_{1}=a \hat{x}$ and $\mathbf{a}_{2}=a \hat{y}$ where $\hat{x}$ and $\hat{y}$ are the base vectors in the spatial domain. Then, the corresponding set of reciprocal lattice base vectors $\left(\mathbf{b}_{1}, \mathbf{b}_{2}\right)$ should be $\left(\frac{2 \pi}{a} \hat{x}, \frac{2 \pi}{a} \hat{y}\right)$ in order to satisfy the reciprocity condition, $a_{i} \cdot b_{j}=2 \pi \delta_{i} j$, where $\delta_{i} j$ is the Kronecker delta function [55]. The reciprocal lattice vectors then, $\mathbf{G}=l \mathbf{b}_{1}+m \mathbf{b}_{2}$ where $l$ and $m$ are integers. Considering the circular (symmetric) PC cylinders with radii, $r$, as in Figure 2(a), the structure factor is found from Eq. (13) as, $S(\mathbf{G})=2 f \frac{J_{1}(G r)}{G r}$, in which the function, $J_{1}($.$) , is$ the first order Bessel function of the first kind. For the rectangular PC case, as in Figure 2(b), the corresponding structure factor is, $S(\mathbf{G})=f \operatorname{sinc}\left(\frac{G_{x} d}{2}\right) \operatorname{sinc}\left(\frac{G_{y} d}{2}\right)$, where $G_{x}$ and $G_{y}$ are the $x$ and $y$-components of the reciprocal lattice vector, G. When the symmetry is reduced in the PC unit-cell by etching an off-centered hole inside the PC rod (see Figure $2(\mathrm{c}))$, the corresponding structure factor transforms into $S(\mathbf{G})=S_{1}(\mathbf{G})-\exp (-i \mathbf{G} \cdot \mathbf{s}) S_{2}(\mathbf{G})$. Here $S_{1}(\mathbf{G})$ and $S_{2}(\mathbf{G})$ are the structure factors for the large (with a radius of $r_{1}$ ) and small (with a radius of $r_{2}$ ) circular PC cylinders encircling the

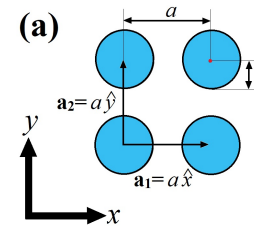

(b)
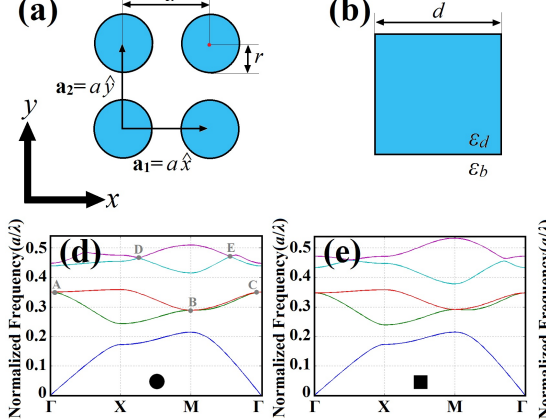
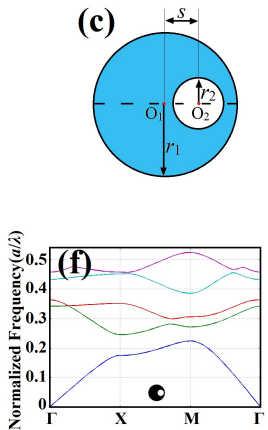

FIG. 2 Geometrical representations of two-dimensional square-lattice PCs composed of a periodic array of dielectric (a) regular (symmetric) cylinders, (b) square cylinders, and (c) $\mathrm{C}_{1}$ symmetric cylinders. The corresponding band diagrams are represented in (d)-(f), respectively. Through the dispersion-relation analyses, the geometrical parameters $r, d, r_{1}$, and $r_{2}$ were set to $0.37 a, 0.66 a, 0.40 a$, and $0.15 a$, respectively.

origin and $\mathbf{s}$ is the shifting vector for a small cylinder of air along the $x$-direction. The dispersion relations of the predetermined three cases are also numerically investigated by using the plane wave expansion method [56] and are shown in Figures 2(d)-2(f). Note that all the band structure calculations and iso-frequency contour (IFC) analyses were conducted by the same method. The geometrical parameters $\mathbf{r}, d, r_{1}$, and $r_{2}$ were set to $0.37 a, 0.66 a, 0.40 a$, and $0.15 a$, respectively, in order to equalize the corresponding filling factors to $f=0.432$. The shifting parameter was fixed at $s=0.20 a$. The corresponding band structures for circular, rectangular, and circular with etched off-center hole PC cylinders were calculated along the $\Gamma-X-M-\Gamma$ direction and are shown in Figures 2(d)-2(f), respectively. The corresponding unit-cell configurations for each case are shown as insets in the same figures. The dielectric PCs were made of silicon, with $\varepsilon_{d}=12$, and the background, as well as the inside of the etched hole, was air, i.e., $\varepsilon_{b}=1$. As can be clearly observed from the dispersion relations for the three cases in Figures 2(d)-2(f), the shapes of first TM bands resemble each other, which means all the configurations behave as a homogeneous media at long wavelengths, so that the effective medium theory holds [57]. However, increasing the frequency (shortening the wavelengths) causes the Bloch modes to be more sensitive to the unit-cell configuration, especially at the edges of Brillouin zone [58]. By comparing the band structures in Figures 2(d)-2(f), it can be seen that reducing the rotational and mirror symmetries (from the circular to circular with off-center hole cases) in the PC unitcell produces higher TM bands, and low-symmetry introduction in PC unit-cell lifts the degeneracies of the optical modes at some points of the Brillouin zone, which are labeled A, B, C, $D$, and $E$ in Figures 2(d). Therefore, one may expect that introducing a symmetry reduction in the unit-cell scale enables the desired dispersion engineering, which will be discussed later in detail.

In the next section, an intriguing phenomenon called Chiral PCs will be discussed and the optical responses of isomers will be compared in the all-dielectric low-symmetric PC case. Under some circumstances, the mirror image of the PC unitcell may become chiral in form. Thus, chirality concept will be mentioned for the manipulation of light. 


\section{WHAT DOES "CHIRALITY" MEAN IN CHEMISTRY AND PHOTONICS?}

Several crystals and molecules exist in nature with a characteristic that even though they may have the same compositions, their physical and chemical properties may differ. In other words, despite having the same molecular formula, some types of compounds may exhibit different characteristics due to different structural arrangements of atoms. This phenomenon is known as isomerism, and compounds that have the same molecular formula but different chemical structures are called isomers [59]. Isomers may have two forms: either structural (constitutional) isomers or stereoisomers. Structural isomers have identical molecular formulas, but different connectivity of atoms. For stereoisomers on the other hand, although the chemical structures and their atomic bonds are the same, they differ in spatial orientation, i.e., the positioning of the crystal compositions in space varies. Moreover, stereoisomers can be subdivided into two types: enantiomers and diastereomers. By definition, enantiomers are stereoisomers for which the mirror-image and the molecule itself cannot be superposed [60]. Examples of well-known enantiomers are chiral molecules, which have the property of handedness (left and right-handed) [61]. The chirality property was discovered by the famous experiment of Louis Pasteur in 1848 [62]. He re-crystallized a salt of tartaric acid and obtained two kinds of crystals, with shapes that were mirrorimages of each other. Thereafter, intensive studies of chiral molecules have revealed that mirror-image isomers, such as chiral crystals, may display different characteristics in terms of optical activity, viz. the optical response of the structures while interacting with light waves [63].

In a similar fashion, when a low-symmetry is introduced to the PC configuration so that the rotational symmetry of the PC unit-cell is broken, then the resulting PC structure and its mirror-image may not be superimposable. These types of low-symmetric PCs may be designated as Chiral PCs. Numerical calculations indicate that Chiral PCs and their images may react differently to light illumination. To be more specific, a basic two-dimensional chiral structure, such as a squarelattice PC configuration with $C_{1}$ symmetry (with no spatialsymmetry), is chosen, and the related dispersion relations as well as IFCs for both the low-symmetric PC and its mirrorimage are compared. These results are presented in Figure 3. As shown in Figure 3(a), the large circular PC cylinder with radius, $r_{1}=0.20 a$, is placed at the origin and the circular rods with small radii, $r_{2}=0.10 a$, are located at the right-side of the larger PC rod. It is important to note that only the rotational symmetry of the proposed Chiral PCs is broken, whereas the translational symmetry is kept intact. In this case, the mirrorimage structure in Figure 3(a) cannot be superimposed on the designed structure with $C_{1}$ symmetry, i.e. with no rotational symmetry, and, therefore, that type of structure can be designated as a Chiral PC. The dispersion diagrams of both square lattice PC configurations are investigated and plotted in Figure $3(b)$. The regarding PC cylinders are composed of silicon, with a dielectric permittivity, $\varepsilon_{d}=12$ and the background is air, i.e., $\varepsilon_{b}=1$. Although the calculated TM bands for both the Chiral PC and its mirror-image overlap at lower frequencies, they start not to coincide with each other at higher bands,
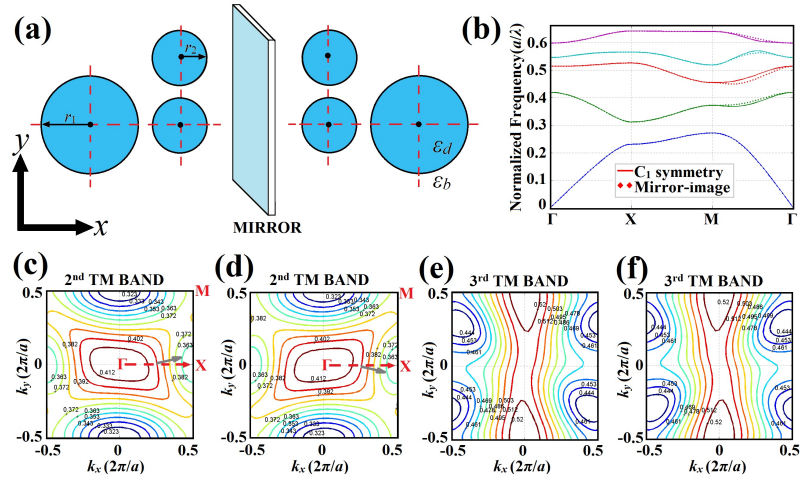

FIG. 3 (a) Illustration of $C_{1}$ symmetric PC configuration (with no spatial symmetry) as chiral PC and its mirror-image isomer. The large circular PC cylinder with radius, $r_{1}=0.20 a$, is placed at the origin and the circular rods with small radii, $r_{2}=0.10 a$, are located at the right-side of the larger PC rod in a primitive cell. The resulting dispersion diagram is calculated and shown in (b). Second and third TM band IFCs for the proposed Chiral PC and its isomer are represented in (c)-(d) and (e)-(f), respectively.

especially along the edge of Brillouin zone, from $\Gamma$ to $M$. In addition, IFCs of the proposed Chiral PCs in Figure 3(a) are analyzed in order to better understand how the optical responses of chiral dielectric PCs differ depending on the direction of wave propagation. As an illustration, the IFCs of second and third TM bands for both the asymmetric chiral PC and its image are calculated and shown in Figures 3(c)-3(f). It is known that the energy flow of light is in the direction perpendicular to the calculated IFCs [64]. Therefore, if the designed square lattice Chiral PC is excited by an incident beam along the $\Gamma-X$ direction, with a centered frequency of $a / \lambda=0.402$ (see Figure $3(\mathrm{c})$ ), the propagating beam starts to deflect, and follows a tilted optical path inside the structure with a positive deflection angle. On the other hand, the incident beam with the same frequency propagates along the $\Gamma-X$ direction inside the mirror-image Chiral PC with a negative deflection angle. Gray arrows have been inserted in Figures 3(c) and 3(d), in order to demonstrate the direction of tilted beam propagation inside the designed Chiral PC and its image. The comparisons of the dispersion relations of designed PC configurations in Figure 3(a) support the chirality idea: In other words, a dielectric PC structure which is not superimposable with its mirrorimage can be considered as a chiral PC, since it may possess dispersive characteristics different from its mirror-image isomer.

There have been conducted numerous studies in literature that investigate PCs with chiral optical properties. Especially, optical activity characteristic has been reported before in different types of materials such as in cholesteric liquid crystals $[65,66]$, photonic metamaterials $[67,68]$ and plasmonic structures [69]. Moreover, circular dichroism effect is also reported in 3D PCs composed of polymeric helices [70, 71] and some potential applications of that chiral optical property are investigated [72]. Plum et al. experimentally verified chiralityinduced negative index of refraction in 3D bilayered metamaterials with four-folded rotational symmetry [73]. Apart from these, there are limited numbers of studies that demonstrate chirality phenomenon in semiconductor-based materials: a 3D rotationally-stacked woodpile PC structure has been 
fabricated and a large optical rotation is observed in such semiconductor-based PCs [74].

There are also relatively few publications that study the effect of low-symmetry on chirality property: In one paper, an efficient polarization control of light is realized by using alldielectric 2D planar PCs with four-fold rotational symmetry [75]. In our study, we numerically proved that keeping the direction of incident light illumination as the same, specifically designed 2D PCs with low-symmetry and its mirror image may optically respond in different manners at certain frequencies (see Figure 3). Chiral PCs either planar 2D or 3D have also been studied for modulating intensity of light and shaping light emission pattern [76, 77]. One other difference between the previously published results and the idea proposed in this article is the way of light interaction with the chiral photonic crystals. Incident light interacts with the chiral device along the out-of-plane direction. On the contrary, we allow light propagation in the plane.

In the following section, several low-symmetric PC studies previously conducted by the authors will be summarized, and based on these studies, some of the potential photonic device applications will be discussed.

\section{POTENTIAL APPLICATIONS BASED ON OUR STUDIES}

There have been an intensive number of studies to investigate all-dielectric two-dimensional PCs that have an intentionally introduced reduced symmetry; to search for their anomalous optical characteristics and to implement these types of PCs in optical device design. In this section, different potential photonic applications are discussed. Firstly, symmetry reduction in PC unit-cells may provide complete PBGs (CPBGs), which occur due to overlapping of TE and TM gaps. Several different approaches have been proposed in the literature [78]-[80]. In one of our previous studies [81], a low-symmetric annular PC structure, called a modified annular PC (MAPC), was designed to obtain larger CPBGs. The designed structure is schematically shown in Figure 4(a). An air hole (or a PC rod) with radius, $r_{2}$, was introduced into the PC rod (or an air hole) having the radius, $r_{1}$, where the background was air (dielectric with the permittivity $\varepsilon_{b}$ ). The inner unit was off-center with a distance parameter, $s$, and rotated with respect to the lattice axis by a rotation angle, $\theta$. Figure 4(b) and 4(c) represent the schematic diagrams of square and triangular MAPC lattices in cases where the background is either air, $\varepsilon_{b}=1$, or $\mathrm{Si}, \varepsilon_{b}=3.45$. In all the configurations, the lattice constant was fixed at $1 a$. The dispersion relations of a symmetric-type annular PC as well as a MAPC with $C_{1}$ symmetry were calculated and are presented in Figure 4(d) and 4(e), in order to better understand the effect of symmetry-reduction. As can be seen in Figure 4(d), CPBG regions do not exist in the symmetric annular PC case; whereas CPBG regions occur in the MAPC case (see Figure 4(e)). Symmetry breaking in the primitive cell lifts the degeneracies in the band structure. As a result, CPBGs appear in the band diagrams [82]. The band gap regions are shaded in Figure 4(e), to clearly show the forbidden frequency intervals. The square lattice MAPC rods with

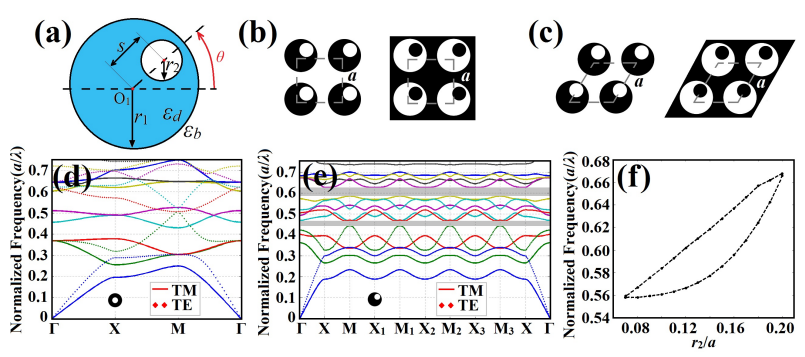

FIC. 4 (a) Geometrical representation of low-symmetric PC unit-cell (MAPC) and corresponding schematic diagrams, for (b) square-lattice and (c) triangular-lattice PCs. Dispersion relations of (d) annular PC and (e) MAPC are shown. TM modes are represented by solid-lines while dashed-lines demonstrate the TE Bloch modes in the figures. For the square lattice MAPCS in air background, the geometrical parameters are set to $\theta=45^{\circ}, r_{1}=0.360 a, r_{2}=0.150 a$, and $s=0.180 a$ while $r_{1}$ and $r_{2}$ are kept the same for the annular PC case. ( $f$ ) Complete PBG map by varying the inner radii of the off-center air-hole, $r_{2} / a$, for the square-lattice MAPC configuration. The corresponding $P C$ unit-cell is shown as an inset in the figure. Reprinted with permission [81], I. H. Giden et al., Appl. Optics 51, 1287-1296 (2012). ( 2012, Optical Society of America.

off-centered air holes having the parameters of, $\theta=45^{\circ}$, $r_{1}=0.360 a, r_{2}=0.150 a$, and $s=0.180 a$, the calculated CPBG width was $\Delta \omega / \omega=7.06 \%$ that lies between $a / \lambda=0.5851$ and $a / \lambda=0.6279$. There also exists a CPBG region lying in the normalized frequency range, $a / \lambda=0.4441-0.4671$, having a CPBG width, $\Delta \omega / \omega=5.05 \%$. A CPBG map is prepared as a function of inner radius and shown in Figure 4(f). The difference between the upper and lower limits of the bands gives the CPBG width, $\triangle \omega$. According to the CPBG map, band gap regions shift upward with increasing inner hole radius, since the resulting filling factor reduces. Moreover, the CPBG around $\Delta \omega / \omega=6.30 \%$ was calculated with the inner hole radius, $r_{2}=0.125 a$. These results support the use of lowsymmetric PCs for polarization-insensitive photonic device applications.

Mode-order conversion applications using low-symmetric PCs have already been studied [83]. For that purpose, a heterostructure is formed by regular (symmetric) PCs and $C_{1}$ symmetric PCs (MAPCs). It should be noted that we fix $\theta=90^{\circ}$ to implement heterostructure investigated in Figure 5. The working principle is that an incident beam is exposed to phase retardation while propagating through the heterostructure. Such a type of phase delay originates from the phase index difference between the conventional PC and MAPC. Figure 5(a) shows the band structures of both the symmetric PC and MAPC when radii of regular PCs are $r=0.36 a$ and geometric parameters of MAPCs are fixed as $\left\{r_{1}, r_{2}, \Delta s\right\}=\{0.40 a, 0.19 a, 0.15 a\}$. The corresponding phase refractive indices are plotted in Figure 5(b). The shaded region in Figure 5(a) designates the frequency interval in which a linear pattern is observed in the second band of the PC and MAPC with respect to the wavevector. By employing the band diagrams shown in Figure 5(a), the corresponding phase indices are obtained depending on normalized frequencies from the formula, $n_{p}=-k / \omega$, where $k=2 \pi / \lambda$. In this expression, the parameters, $k$ and $\omega$, represent the relevant wavevector and angular frequency, respectively. The corresponding phase indices for both symmetric and low-symmetric PCs were calculated and are demonstrated in 


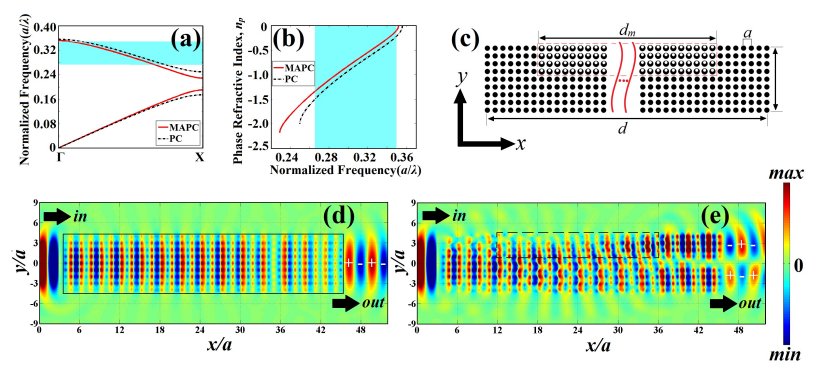

FIG. 5 (a) Dispersion relations and (b) corresponding phase index distributions of square-lattice MAPC (solid-red line) and PC (dashed-black line) structures. In bandstructure calculations, radii of regular PCs are $r=0.36 a$ and geometric parameters of MAPCs are fixed as $\left\{r_{1}, r_{2}, \Delta s\right\}=\{0.40 a, 0.19 a, 0.15 a\}$. (c) Schematic view of dielectric $P C$ heterostructure design for the even-to-odd mode-conversion application. The longitudinal lengths of MAPCs, $d_{m}$, and the heterostructure, $d$, were adjusted to $15 a$ and $25 a$, respectively, in order to achieve a $\pi$-phase shift at the output channel. The calculated steady-state electric-field distributions of (d) regular square-lattice PCs and (e) PC heterostructure at the operating frequency, $a / \lambda=0.325$. Reprinted with permission [83], N. Erim et al., J. Opt. Soc. Am. B 30, 3086-3094 (2013). (c2013, 0ptical Society of America.

Figure 5(b). Their values are negative for the second bands because the corresponding frequencies in the second TM bands decrease depending on the increasing propagation constant. A nearly constant phase refractive index difference, $\Delta n_{p}=0.0634$, was calculated in a broad frequency interval, $a / \lambda=0.275-0.350$, which is represented by the shaded region in Figure 5(b). This type of phase index difference causes the propagating light to travel at different phase velocities and, hence, different optical path lengths occur inside the heterostructure. Therefore, a $\pi$-phase shift can be achieved at the output by selecting the length of the MAPC region, which is given as $d_{m}$ in Figure 5(c). The required length, $d_{m}$, can be calculated by the following expression, $\Delta \phi=\Delta n_{p} \cdot k(\lambda) \cdot d_{m}$, where $\Delta \phi$ represents the output phase shift. In our design, the longitudinal lengths of MAPCs, $d_{m}$, and the heterostructure, $d$, were adjusted to $15 a$ and $25 a$, respectively, in order to obtain a $\pi$-phase shift at the output channel. The width of the structure, $w$, was set to $8 a$. Finite difference time domain (FDTD) analyses were also conducted to investigate how low-symmetric PCs affect the shape of propagating beams' mode profiles [84]. Figures 5(d) and 5(e) compare the beam propagation inside regular PCs and the designed heterostructure. The structures were excited by a guided fundamental TM mode $\left(\mathrm{TM}_{0}\right)$, with a center frequency fixed at $a / \lambda=0.325$. The term "mode" means the field amplitude shape of allowed modes $\left(E_{z}\right.$ component for TM modes) inside the structure. As can be seen from the steady-state field distribution in Figure 5(d), the incident beam still propagates with a fundamental $\mathrm{TM}_{0}$ mode profile, despite reaching the end of the regular PC structure. On the other hand, the propagating beam in the designed PC heterostructure is exposed to $\pi$-phase difference at the end; Figure 5(e) represents a snapshot of this beams electric field. The FDTD results indicate that the fundamental even mode profile $\left(\mathrm{TM}_{0}\right)$ can be efficiently converted into the odd $\left(\mathrm{TM}_{1}\right)$ mode or to higher order TM modes, just by introducing a low-symmetric PC region inside the regular PC configuration.
A non-diffracting dispersive effect called self-collimation or, sometimes, super-collimation and its various optical applications have been studied intensively [85]-[87]. Such a dispersive property enables guiding of light, while propagating inside a PC structure, without significant diffraction. Super-collimation characteristic have already been investigated for low symmetric PCs over a broad bandwidth [88]. $\mathrm{A} \mathrm{C}_{4}$ symmetric PC is proposed in order to achieve supercollimation over a wide frequency interval. The designed two-dimensional PC configuration is called a star-shaped PC (STAR-PC). Low-symmetry was introduced in the scale of PC unit-cell and the resulting primitive cell is presented in Figure 6(a). The proposed configuration has four vertices and eight edges. The width of each edge is denoted by $w$ and internal angle, $\theta$, of each vertex is fixed at $\theta=45^{\circ}$. The width of the edges was fixed at $w=0.30 a$ and, thus, the resulting filling factor was $f=3 \cdot w^{2}=0.27$. The investigated PC cylinders were composed of silicon, $\varepsilon_{d}=12$ and the background was air, i.e., $\varepsilon_{b}=1$. Reduction of rotational symmetry in the unit-cell has significant effects on the dispersion characteristics of PCs, especially at higher bands. To better understand the effect of low-symmetry on the dispersion relations of PCs, the equi-frequency contours (EFCs) of both a $C_{4}$ symmetric STAR-PC and a symmetric PC were calculated, with the same filling factor and corresponding EFCs at the fifth TM band, and are shown in Figures 6(b) and 6(c), respectively. Linear sections in the curves appear for the low-symmetric PC case, which is shaded in Figure 6(b), and, therefore, that bring about a strong super-collimation property over a large bandwidth, $\Delta \omega=16.42 \%$, in the frequency range, $a / \lambda=[0.492,0.580]$. On the other hand, as can be seen in Figure 6(c), the regular PC has flat contours in the frequency interval, $a / \lambda=[0.577,0.581]$, and, thus, the available bandwidth is $\Delta \omega=0.69 \%$, which is very small compared to the STAR-PC case. FDTD analyses have also been conducted in order to observe the beam evolution inside the designed structure. For this reason, a continuous TM polarized beam was propagated in air and in the STAR-PC, and their steady-state electric field patterns are depicted in Figures 6(d) and 6(e), respectively. Dashed lines in the figures represent the envelope of the propagating beam. Moreover, arrows inserted in the figures indicate the direction of source-illumination. The width of the beam is $11 a$ and the operating frequency is $a / \lambda=0.540$. As can be clearly seen in Figures 6(d) and 6(e), after propagation over a distance, $L=200 a$, there is no observable spatial broadening in the STAR-PC configuration, whereas a significant spreading of beam is observed in air. The calculated results show that by means of low-symmetric PCs, one may realize super-collimation based single-mode optical devices, such as optical interconnects and routers, without substantial light diffraction.

As we discussed previously, breaking the structural symmetry in the unit-cell causes dramatic effects on the dispersion characteristics. In the dispersion diagram for low symmetry structure, the first band behaves like an isotropic medium for lower frequencies. However, for higher bands, considerably different EFCs occur due to the lack of symmetry in the PC unit-cell, as can be seen in Figure 6(b). It is important to note that the higher frequencies are influenced strongly by the symmetry 

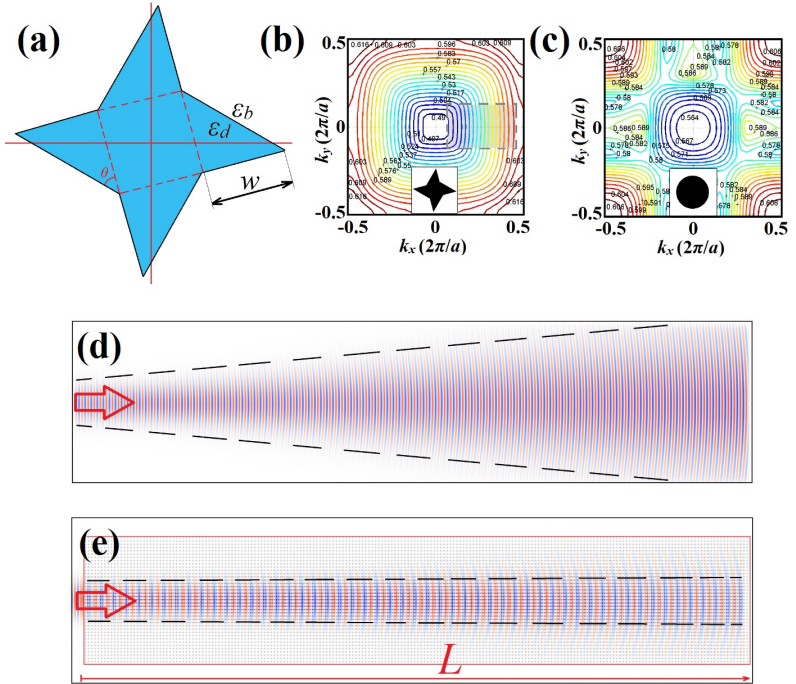

FIG. 6 (a) Geometrical representation of low-symmetric STAR-PC unit-cell with its structural parameters. IFCS of the fifth TM band for square lattice (b) STAR PC and (c) regular (symmetric) PC configurations. The width of the STAR-PC edges is fixed at $w=0.30 a$. The shaded region in (b) represents the frequency interval in which the contours appear as linear (flat) curves. Steady-state electric-field patterns of a continuous beam that propagates in (d) free space and (e) designed STAR PC structure. Dashed lines in the figures indicate the envelope of propagating beam. The incident beam operates at the normalized frequency, $a / \lambda=0.540$. Reprinted with permission [88], I. H. Giden et al., Photonics and Nanostructures - Fundamentals and Applications 11, 132-138 (2013). (c) 2013, Elsevier.

of the primitive PC cell. Moreover, even a rotational operation on the PC unit-cell provides intriguing characteristics, such as wavelength selectivity [89]. The proposed structure is shown in Figure 6(a), and its geometrical parameters are represented in Figure $7(\mathrm{a})$. This structure was rotated through $45^{\circ}$ in a clockwise direction. The resulting structural parameters (filling factor, internal angle, dielectric permittivity, and air background) were kept the same as the structure in Figure 6(a). The rotation of the symmetric PC structure in $C_{4}$ caused tilting of the IFCs at the fifth TM band, which is presented in Figure 7(b). As can be seen in that figure, the amount of tilt in the nearly flat EFCs (self-collimation contours) increases with the normalized frequency. Therefore, a light beam can follow different paths (directions) inside the periodic structure due to the tilting self-collimating effect. This property may allow for the spatial resolution of different wavelengths at the output of the PC structure. The EFCs for the frequencies at which the wavelength selectivity behavior appears are presented in Figure 7(c), and the corresponding Brillouin zone of the STAR$\mathrm{PC}$ is shown as an inset in the figure. The corresponding frequency contours are selected from the fifth band EFCs in Figure $7(\mathrm{c})$, and lie between $a / \lambda=0.520$ and $a / \lambda=0.568$, with a broad bandwidth, $8.82 \%$. Numerical analyses have also been conducted in order to examine the wavelength division behavior of the STAR-PCs. For this reason, a STAR-PC was sequentially illuminated by a continuous TM polarized beam, and Figure 7(d) shows their steady-state electric field patterns at three selected wavelengths $\left(\lambda_{1}=1621.5 \mathrm{~nm}, \lambda_{2}=1550 \mathrm{~nm}\right.$ and $\lambda_{3}=1484.5 \mathrm{~nm}$ ) within the region of interest. The cascaded slices taken at the output of the field distributions are shown to demonstrate the spatial shifts of the output signal. The spatial separation of output signals in terms of their wave-
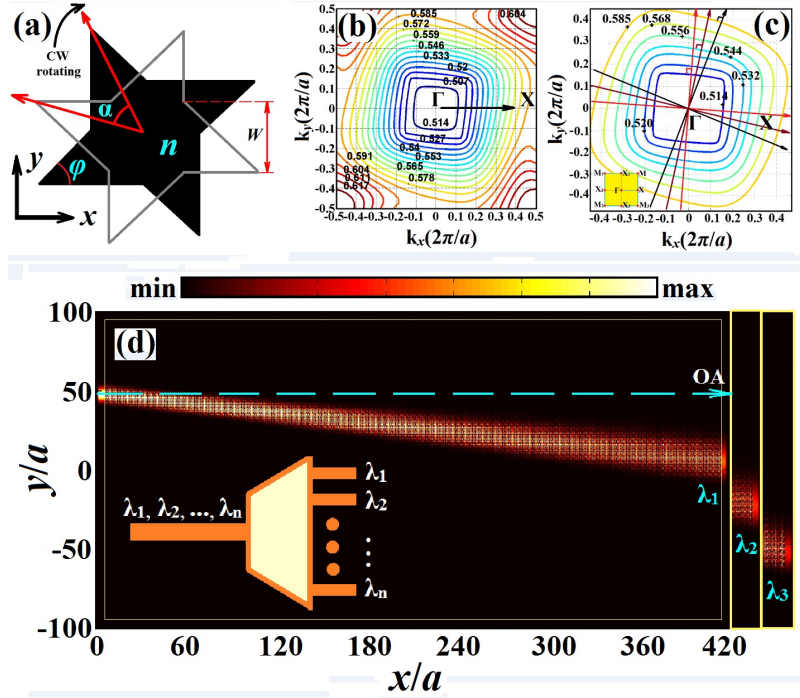

FIG. 7 (a) Unit-cell of proposed STAR-PC. It is composed of a dielectric material (Si with a refractive index of 3.45) in an air background. Calculated EFCS of the fifth TM-band of the square lattice (b) STAR-PCS and (c) detailed representation of the same band EFCS for the selected frequencies. In this case, the width of the STAR-PC edges is fixed at $w=0.30 a$. (d) The spatial distribution of electric field intensity for the beam propagating in the tilted self-collimated manner inside the square lattice STAR-PC. Inset shows illustration of $n$ channel wavelength selective device. Reprinted with permission [89], M. Turduev et al., Photonics and Nanostructures - Fundamentals and Applications 11, 241-252 (2013). (c2013, Elsevier.

lengths is accomplished by means of diffraction-free beam propagation (tilted self-collimation). By this means, sufficient spatial shifts can be introduced between each channel, since the spatial beam profile is almost preserved as it propagates inside the structure. This phenomenon increases the potential of STAR-PCs as a wavelength selective medium. To better understand the bandwidth enhancement for super-collimation, the role of the order of the symmetry reduction will be further explored in the future.

Other type of low-symmetric unit cells can produce supercollimation and wavelength selectivity. On the other hand, bandwidth and wavelength sensitivity may change from structure to structure. Since these properties appear at relatively high band, feature sizes in terms of sharp corners enhance the observed physical characteristics.

Apart from the above-mentioned applications, by using the intrinsic dispersive characteristics of the low-symmetric PCs, different types of photonic devices such as beam routers, splitters, and deflectors can be realized [90]. Moreover, polarization-independent waveguide design can be implemented by PCs with reduced-symmetry [91].

\section{FUTURE EXPECTATIONS AND CONCLUSION}

In this review paper, we investigated the effect of symmetryreduction on the dispersive characteristics of all-dielectric PCs. Breaking the rotational symmetry of a PC unit-cell produces various anomalous optical characteristics such as complete PBG, tilted self-collimation, super-collimation, and 
wavelength selectivity. Besides, the low symmetric unit-cell provides additional parameters to control the dispersive features of Bloch modes. Furthermore, the symmetry-reduction in PC unit-cell scale produces a novel feature named "chirality", which has not been thoroughly studied for all-dielectric PCs. Using such intrinsic properties of low-symmetric PCs, it is feasible to design a variety of photonic integrated devices such as polarization-insensitive waveguides, beam routers/deflectors, splitters, and wavelength demultiplexers. Moreover, it is important to investigate these types of photonic media in the presence of point or line defects. Resonance modes in the cavities can be manipulated by the reduced symmetry in a PC unit-cell. Dispersion management of waveguide modes may uniquely sustain slow light propagation.

As a future goal, we intend to study the symmetryreduction in PCs by rearranging the lattice periodicity into quasi-periodic and disordered lattices. Moreover, we will investigate the cavity and resonator effects in low-symmetric PCs by introducing intentional perturbations (point and line-defects) inside the all-dielectric periodic media. We also plan to conduct detailed investigations of all-dielectric chiral PCs. Further investigations are required to fully exploit the potential of symmetry reduction and the chirality concept in photonic crystals.

\section{ACKNOWLEDGEMENTS}

HK acknowledges partial support of the Turkish Academy of Science.

\section{References}

[1] L. Rayleigh, "On the maintenance of vibrations by forces of double frequency, and on the propagation of waves through a medium endowed with a periodic structure," Phil. Mag. S. 5 24(147), 145-159 (1887).

[2] A. Yariv, and P. Yeh, Photonics: Optical Electronics in Modern Communications (0xford University Press, 0xford, 2007).

[3] E. Yablonovitch, "Inhibited spontaneous emission in solid-state physics and electronics," Phys. Rev. Lett. 58, 2059-2062 (1987).

[4] S. John, "Strong localization of photons in certain disordered dielectric superlattices," Phys. Rev. Lett. 58(23), 2486-2489 (1987).

[5] J. D. Joannopoulos, S. G. Johnson, J. N. Winn, and R. D. Meade, Photonic Crystals: Molding the Flow of Light, (Princeton University Press, New Jersey, 2008).

[6] Y. Akahane, T. Asano, B-S. Song, and S. Noda, "High-Q photonic nanocavity in a two-dimensional photonic crystal," Nature 425 , 944-947 (2003).

[7] B. S. Song, S. Noda, T. Asano, and Y. Akahane, "Ultra-high-Q photonic double-heterostructure nanocavity," Nat. Mater. 4, 207-210 (2005).

[8] L. Wu, M. Mazilu, T. Karle, and T. F. Krauss, "Superprism phenomena in planar photonic crystals," IEEE J. Quantum Elect. 38, 915-918 (2002).

[9] H. Kosaka, T. Kawashima, A. Tomita, M. Notomi, T. Tamamura, T. Sato, and S. Kawakami, "Self-collimating phenomena in pho- tonic crystals," Appl. Phys. Lett. 74, 1212-1214 (1999).

[10] E. Chow, A. Grot, L. W. Mirkarimi, M. Sigalas, and G. Girolami, "Ultracompact biochemical sensor built with two-dimensional photonic crystal microcavity," Opt. Lett. 29, 1093-1095 (2004).

[11] H. Kurt, M. N. Erim, and N. Erim, "Various photonic crystal biosensor configurations based on optical surface modes," Sensor. Actuat. B-Chem. 165(1), 68-75 (2012).

[12] T. F. Krauss, R. M. D. L. Rue, and S. Brand, "Two-dimensional photonic-bandgap structures operating at near infrared wavelengths," Nature 383(6602), 699-702 (1996).

[13] 0. Painter, R. K. Lee, A. Scherer, A. Yariv, J. D. 0’Brien, P. D. Dapkus, and L. Kim, "Two-dimensional photonic band-gap defect mode laser," Science 284, 1819-1821 (1999).

[14] S. Noda, A. Chutinan, and M. Imada, "Trapping and emission of photons by a single defect in a photonic bandgap structure," $\mathrm{Na}$ ture 407, 606-610 (2000).

[15] H. Kurt, "Theoretical study of directional emission enhancement from photonic crystal waveguides with tapered exits," IEEE Photonic. Tech. L. 20, 1682-1684 (2008).

[16] A. E. Akosman, M. Mutlu, H. Kurt, and E. Ozbay, "Compact wavelength de-multiplexer design using slow light regime of photonic crystal waveguides," Opt. Express 19, 24129-24138 (2011).

[17] T. Gorishnyy, C. K. Ullal, M. Maldovan, G. Fytas, and E. L. Thomas, "Hypersonic Phononic Crystals," Phys. Rev. Lett. 94, 115501 (2005).

[18] A. Khelif, A. Choujaa, S. Benchabane, B. Djafari-Rouhani, and V. Laude, "Guiding and bending of acoustic Waves in Highly Confined Phononic Crystal Waveguides," Appl. Phys. Lett. 84(22), 4400-4402 (2004).

[19] S. Yang, J. H. Page, Z. Liu, M. L. Cowan, C. T. Chan, and P. Sheng, "Focusing of Sound in a 3D Phononic Crystal," Phys. Rev. Lett. 93(2), 024301 (2004).

[20] V. Romero-Garcia, R. Pico, A. Cebrecos, V. J. Sanchez-Morcillo, and K. Staliunas, "Enhancement of sound in chirped sonic crystals," Appl. Phys. Let. 102, 091906 (2013).

[21] D. S. Wiersma, "Disordered photonics," Nat. Photonics 7, 188-196 (2013).

[22] E. R. Martins, J.T. Li, Y. K. Liu, V. Depauw, Z. X. Chen, J. Y. Zhou, and T. F. Krauss, "Deterministic quasi-random nanostructures for photon control," Nat. Commun. 4, 2665 (2013).

[23] M. Segev, Y. Silberberg, and D. N. Christodoulides, "Anderson Iocalization of light," Nat. Photonics 7, 197-204 (2013).

[24] B. Redding, S. F. Liew, R. Sarma, and H. Cao, "Compact spectrometer based on a disordered photonic chip," Nat. Photonics 7(9), 746-751 (2013).

[25] H. Cao, Y. G. Zhao, S. T. Ho, E. W. Seelig, Q. H. Wang, and R. P. H. Chang, "Random Laser Action in Semiconductor Powder," Phys. Rev. Lett. 82, 2278-2281 (1999).

[26] S. Gottardo, R. Sapienza, P. D. García, A. Blanco, D. S. Wiersma, and C. López, "Resonance-driven random lasing," Nat. Photonics 2, 429-432 (2008).

[27] N. M. Lawandy, "Disordered media: Coherent random lasing," Nat. Phys. 6, 246-248 (2010).

[28] V. Roppo, D. Dumay, J. Trull, C. Cojocaru, S. M. Saltiel, K. Staliunas, R. Vilaseca, et al., "Planar second-harmonic generation with noncollinear pumps in disordered media," Opt. Express 16, 14192-14199 (2008). 
[29] Z. V. Vardeny, A. Nahata, and A. Agrawal, "Optics of photonic quasicrystals," Nat. Photonics 7, 177-187 (2013).

[30] M. E. Zoorob, M. D. B. Charlton, G. J. Parker, J. J. Baumberg, and M. C. Netti, "Complete photonic bandgaps in 12-fold symmetric quasicrystals," Nature 404, 740-743 (2000).

[31] N. D. Lai, J. H. Lin, Y. Y. Huang, and C. C. Hsu, "Fabrication of twoand three-dimensional quasi-periodic structures with 12-fold symmetry by interference technique," Opt. Express 14, 10746-10752 (2006).

[32] A. Della Villa, S. Enoch, G. Tayeb, V. Pierro, V. Galdi, and F. Capolino, "Band gap formation and multiple scattering in photonic quasicrystals with a Penrose-type Lattice," Phys. Rev. Lett. 94, 183903 (2005).

[33] M. A. Kaliteevski, S. Brand, R. A. Abram, T. F. Krauss, R. M. De La Rue, and P. Millar, "Two-dimensional Penrose-tiled photonic quasicrystals: diffraction of light and fractal density of modes," J. Mod. Optic. 47(11), 1771-1778 (2000)

[34] Y. A. Vlasov, M. I. Kaliteevski, and V. V. Nikolaev, "Different regimes of light localization in a disordered photonic crystal," Phys. Rev. B. 60, 1555-1562 (1999).

[35] M. Werchner, M. Schafer, M. Kira, S. W. Koch, J. Sweet, J. D. Olitzky, J. Hendrickson, et al., "One dimensional resonant Fibonacci quasicrystals: noncanonical linear and canonical nonlinear effects," Opt. Express 17, 6813-6828 (2009).

[36] W. Gellermann, M. Kohmoto, B. Sutherland, and P. C. Taylor, "Localization of light waves in Fibonacci dielectric multilayers," Phys. Rev. Lett. 72, 633-636 (1994).

[37] Y. S. Chan, C. T. Chan, and Z. Y. Liu, "Photonic Band Caps in Two Dimensional Photonic Quasicrystals," Phys. Rev. Lett. 80, 956-959 (1998).

[38] M. C. Rechtsman, H.-.C Jeong, P. M. Chaikin, S. Torquato, and P. J. Steinhardt, "Optimized Structures for Photonic Quasicrystals," Phys. Rev. Lett. 101, 073902 (2008).

[39] M. Florescu, S. Torquato, and P. J. Steinhardt, "Complete band gaps in two-dimensional photonic quasicrystals," Phys. Rev. B 80, 155112 (2009).

[40] W. Man, M. Megens, P. J. Steinhardt, and P. M. Chaikin, "Experimental measurement of the photonic properties of icosahedral quasicrystals," Nature 436, 993-996 (2005).

[41] J. Hung Lin, W. L. Chang, H-Y. Lin, T-H. Chou, H-C. Kan, and C. C. Hsu, "Enhancing light extraction efficiency of polymer lightemitting diodes with a 12-fold photonic quasi crystal," Opt. Express 21, 22090-22097 (2013).

[42] C. Kittle, Introduction to solid state physics (John Wiley a Sons, New York, 1996).

[43] Q. Gong, and X. Hu, Photonic Crystals: Principle and Applications (Pan Stanford Publishing, Singapore, 2012).

[44] Z-Y. Li, B-Y Gu, and G-Z Yang, "Large absolute band gap in 2D anisotropic Photonic crystals," Phys. Rev. Lett. 81, 2574-2577 (1998).

[45] C. M. Anderson, and K. P. Giapis, "Larger two-dimensional Photonic band gaps," Phys. Rev. Lett. 77, 2949-2952 (1996).

[46] X. Zhang, and Z-Q Zhang, "Creating a gap without symmetry breaking in two-dimensional photonic crystals," Phys. Rev. B 61, 9847-9850 (2000).

[47] N. Susa, "Large absolute and polarization-independent photonic band gaps for various lattice structures and rod shapes," J. Appl.
Phys. 91, 3501-3510 (2002).

[48] M. Agio, and L. C. Andreani, "Complete photonic band gap in a two-dimensional chessboard lattice," Phys. Rev. B 61, 15519-15522 (2000).

[49] R. Wang, X-H. Wang, B-Y. Gu, and G-Z. Yang, "Effects of shapes and orientations of scatterers and lattice symmetries on the photonic band gap in two-dimensional photonic crystals," J. Appl. Phys. 90, 4307-4313 (2001).

[50] T. F. Khalkhali, B. Rezaei, and M. Kalafi, "Enlargement of absolute photonic band gap in modified 2D anisotropic annular photonic crystals," Opt. Commun. 284(13), 3315-3322 (2011).

[51] P. Shi, K. Huang, and Y-P. Li, "Photonic crystal with complex unit cell for large complete band gap," Opt. Commun. 285(13), 3128-3132 (2012).

[52] K. Sakoda, Optical Properties of Photonic Crystals (Springer-Verlag, Berlin, 2005).

[53] S. H. Moosavi Mehr, and S. Khorasani, "Influence of asymmetry on the band structure of photonic crystals," Proc. SPIE 7609, $76091 \mathrm{C}$ (2010).

[54] P. G. Luan, Z. Ye, "Two dimensional photonic crystals," arXiv:condmat/0105428 (2001)

[55] J. D. Joannopoulos, S. G. Johnson, J. N. Winn, and R. D. Meade, Photonic Crystals (Princeton University Press, Princeton, 2008).

[56] S. Johnson, and J. Joannopoulos, "Block-iterative frequencydomain methods for Maxwell's equations in a planewave basis," Opt. Express 8, 173-190 (2001).

[57] D. E. Aspnes, "Local-field effects and effective-medium theory: A microscopic perspective," Am. J. Phys. 50, 704-709 (1982).

[58] Y. Kurosaka, S. Iwahashi, K. Sakai, E. Miyai, W. Kunishi, D. Ohnishi, and S. Noda, "Band structure observation of 2D photonic crystal with various $V$-shaped air-hole arrangements," IEICE Electron. Expr. 6, 966-971 (2009).

[59] R. H. Petrucci, R. S. Harwood, and F. G. Herring, General Chemistry: Principles and Modern Applications (Pearson Prentice Hall, New Jersey, 2002).

[60] E. L. Eliel and S. H. Wilen, Stereochemistry of Organic Compounds (Wiley, Chichester, 1994).

[61] A. D. McNaught and A. Wilkinson, Compendium of Chemical Terminology (Blackwell Scientific Publications, 0xford 1997).

[62] L. Pasteur, Researches on the molecular asymmetry of natural organic products (1848). English translation of French original, published by Alembic Club Reprints (Vol. 14, pp. 1-46) in 1905, facsimile reproduction by SPIE in a 1990 book.

[63] L. D. Barron, Molecular Light Scattering and Optical Activity (Cambridge University Press, Cambridge, 2004).

[64] P. Yeh, "Electromagnetic propagation in birefringent layered media," J. Opt. Soc. Am. 69, 742-756 (1979).

[65] H.-S. Kitzerow, and C. Bahr (eds.), Chirality in Liquid Crystals (Springer, New York, 2001).

[66] A. H. Gevorgyan, "Optical properties of a stack of right- and lefthanded layers of a cholesteric liquid crystal," Opt. Spectrosc. 113, 141-152 (2012).

[67] J. B. Pendry, "A chiral route to negative refraction," Science 306, 1353-1355 (2004).

[68] Z. Li and M. Mutlu, and E. Ozbay, "Chiral metamaterials: from optical activity and negative refractive index to asymmetric transmission," J. Opt. 15(2), 023001 (2013). 
[69] M. Hentschel, M. Schoferling, T. Weiss, N. Liu, and H. Giessen, "Three dimensional chiral plasmonic oligomers," Nano Lett. 12, 2542-2547 (2012).

[70] M. Thiel, H. Fischer, G. von Freymann, and M. Wegener, "Threedimensional chiral photonic superlattices," Opt. Lett. 35, 166-168 (2010).

[71] M. Thiel, G. von Freymann, and M. Wegener, "Layer-by-layer threedimensional chiral photonic crystal," Opt. Lett. 32, 2547-2549 (2007).

[72] M. Thiel, M. Decker, M. Deubel, M. Wegener, S. Linden, and G. von Freymann, "Polarization stop bands in chiral polymeric three-dimensional photonic crystals," Adv. Mater. 19, 207-210 (2007).

[73] E. Plum, J. Zhou, J. Dong, V. A. Fedotov,T. Koschny, C. M. Soukoulis, and N. I. Zheludev, "Metamaterial with negative index due to chirality," Phys. Rev. B 79, 035407 (2009).

[74] S. Takahashi, A. Tandaechnurat, R. Igusa, Y. Ota, J. Tatebayashi, S. Iwamoto, and Y. Arakawa, "Giant optical rotation in a threedimensional semiconductor chiral photonic crystal," Opt. Express 21, 29905-29913 (2013).

[75] K. Konishi, B. Bai, X. Meng, P. Karvinen, J. Turunen, Y. P. Svirko, and M. Kuwata-Conokami, "Observation of extraordinary optical activity in planar chiral photonic crystals," Opt. Express 16, 7189-7196 (2008).

[76] W. Zhang, A. Potts, A. Papakostas, and D. M. Bagnall, "Intensity modulation and polarization rotation of visible light by dielectric planar chiral metamaterials," Appl. Phys. Lett. 86, 231905 (2005).

[77] K. Konishi, M. Nomura, N. Kumagai, S. Iwamoto, Y. Arakawa, and M. Kuwata-Conokami, "Circularly polarized light emission from semiconductor planar chiral nanostructures," Phys. Rev. Lett. 106, 057402 (2011).

[78] X. Zhu, Y. Zhang, D. Chandra, S.-C. Cheng, J. M. Kikkawa, and S. Yang, "Twodimensional photonic crystals with anisotropic unit cells imprinted from poly(dimethylsiloxane) membranes under elastic deformation," Appl. Phys. Lett. 93, 161911 (2008).

[79] B. Rezaei and M. Kalafi, "Engineering absolute band gap in anisotropic hexagonal photonic crystals," Opt. Commun. 266, 159-163 (2006).

[80] J. Feng, Y. Chen, J. Blair, H. Kurt, R. Hao, D. S. Citrin, C. J. Summers, et al., "Fabrication of annular photonic crystals by atomic layer deposition and sacrificial etching," J. Vac. Sci. Technol. B 27, 568-572 (2009).
[81] I. H. Giden and H. Kurt, "Modified annular photonic crystals for enhanced band gap properties and iso-frequency contour engineering," Appl. Optics 51, 1287-1296 (2012).

[82] K. M. Ho, C.T. Chan, and C.M. Soukoulis, "Existence of a photonic gap in periodic dielectric structures," Phys. Rev. Lett. 65, 3152 (1990).

[83] N. Erim, I. H. Giden, M. Turduev, and H. Kurt, "Efficient mode-order conversion using a photonic crystal structure with low symmetry," J. Opt. Soc. Am. B 30, 3086-3094 (2013).

[84] A. Taflove and S. C. Hagness, Computational Electrodynamics: The Finite-Difference Time-Domain Method(Artech House, Massachusetts, 2005).

[85] H. Kosaka, T. Kawashima, A. Tomita, M. Notomi, T. Tamamura, T. Sato, and S. Kawakami, "Self-collimating phenomena in photonic crystals," Appl. Phys. Lett. 74, 1212-1214 (1999).

[86] R. E. Hamam, M. Ibanescu, S.G. Johnson, J.D. Joannopoulos, and M. Soljacić, "Broadband super-collimation in a hybrid photonic crystal structure," Opt. Express 17, 8109-8118 (2009).

[87] X. Yu and S. Fan, "Bends and splitters for self-collimated beams in photonic crystals," Appl. Phys. Lett. 83, 3251-3253 (2003).

[88] I. H. Giden, M. Turduev, and H. Kurt, "Broadband super-collimation with low-symmetric photonic crystal," Photonic. Nanostruct. 11, 132-138 (2013).

[89] M. Turduev, I. H. Giden, and H. Kurt, "Extraordinary wavelength dependence of self-collimation effect in photonic crystal with low structural symmetry," Photonic. Nanostruct. 11, 241-252 (2013).

[90] H. Kurt, M. Turduev, and I. H. Giden, "Crescent shaped dielectric periodic structure for light manipulation," Opt. Express 20, 7184-7194 (2012).

[91] M. Turduev, I. H. Giden, and H. Kurt, "Modified annular photonic crystals with enhanced dispersion relations: polarization insensitive self-collimation and nanophotonic wire waveguide designs," J. Opt. Soc. Am. B 29, 1589-1598 (2012). 